\title{
Retroviral Vector Biosafety: Lessons from Sheep
}

\author{
Anne Van den Broeke* and Arsène Burny \\ Laboratory of Experimental Hematology, Bordet Institute, 1000 Brussels, Belgium
}

Received 6 June 2002; accepted 19 July 2002

\begin{abstract}
The safety of retroviral-based systems and the possible transmission of replication-competent virus to patients is a major concern associated with using retroviral vectors for gene therapy. While much effort has been put into the design of safe retroviral production methods and effective in vitro monitoring assays, there is little data evaluating the risks resulting from retroviral vector instability at post-transduction stages especially following in vivo gene delivery. Here, we briefly describe and discuss our observations in an in vivo experimental model based on the inoculation of retroviral vector-transduced tumor cells in sheep. Our data indicates that the in vivo generation of mosaic viruses is a dynamic process and that virus variants, generated by retroviral vector-mediated recombination, may be stored and persist in infected individuals prior to selection at the level of replication. Recombination may not only restore essential viral functions or provide selective advantages in a changing environment but also reestablish or enhance the pathogenic potential of the particular virus undergoing recombination. These observations in sheep break new ground in our understanding of how retroviral vectors may have an impact on the course of a preestablished disease or reactivate dormant or endogenous viruses. The in vivo aspects of vector stability raise important biosafety issues for the future development of safe retroviral vector-based gene therapy.
\end{abstract}

Vectors derived from retroviruses are the most extensively used vehicles for gene transfer (reviewed by $\mathrm{Hu}$ and Pathak [1]). In this respect, retroviral vectors display a number of unique features including the absence of viral gene expression and the capacity to integrate into the host genome, a prerequisite for permanent gene transfer in a number of applications. The safety of retroviralbased systems, however, and the possible transmission of replication-competent virus to patients is a major concern associated with using retroviral vectors for gene therapy.

It is well documented that retroviruses display high recombination and mutation rates $[2,3]$. Replicationcompetent viruses (RCR) can be generated through recombination between the vector and either the helper constructs required for vector production or endogenous retroviruses present in the host genome. Both the host cell derived RNA polymerase II and the virus-encoded reverse transcriptase are incapable of exerting exonucleolytic proofreading activity and therefore contribute to the generation of genetically divergent retroviruses. The dimeric nature of the genome moreover allows template switching of reverse transcriptase during the copying of copackaged RNA molecules, leading to the generation of recombinant proviruses harboring information derived from both parental RNAs. For further information regarding the basic concepts of recombination, examples of recombinational reassortment and their impact on retrovirus evolution, we refer the reader to the reviews of $\mathrm{Ne}$ groni and Buc [4] and Mikkelsen and Pedersen [5].

Because of these safety concerns, screening assays have been elaborated for testing the presence of RCR during production of retroviral vectors, in ex vivo-transduced cells and during patient follow-up $[6,7]$. Much effort has been put into the design of safe retroviral vector production methods for generating helper free recombinant vectors through the development of systems with reduced homology between retroviral vector and packaging components, self-inactivating vectors, or split-genome conditional packaging systems $[8,9,10]$. Cell culture studies have provided examples of retroviral genetic changes introduced by recombination and there is extensive data demonstrating the impact of recombination during vector preparation $[5,11,12,13]$. However, in vitro approaches currently used to monitor structural changes introduced in the integrated vector post-transduction are limited by the absence of host-associated processes that govern genetic changes and therefore do not fully mimic the complex in vivo situation.

There is very little data evaluating the dynamics of provirus structural modifications in vivo, and the risks resulting from retroviral vector instability at post-transduction stages are largely ignored, especially following in vivo gene delivery. The generation of mosaic retroviruses has been described in mice and primates $[14,15,16]$, but to our knowledge, there is poor direct evidence of in vivo recombination that involves retroviral vector sequences and either exogenous or endogenous retroviruses. $\mathrm{Hu}-$ man endogenous retroviruses (HERVs) are the proviral remnants of previous retroviral infections and make up as much as $8 \%$ of the human genome [17]. Most importantly, some endogenous retroviruses are capable of interacting with exogenous counterparts, including retroviral 
vectors, through different mechanisms including recombination and transactivation [18]. Endogenous retroelements thus represent a significant source of functional viral sequences, which may serve as donors in recombination. In addition, proteins-provided in trans by retroviral vectors may lead to the spread of otherwise inactive endogenous retroviruses. This phenomenon may challenge the safe usage of retroviral vector-based gene vehicles.

Although recombination between MLV-derived vectors and human endogenous retroviruses may thus potentially happen, there are no examples of in vivo crossspecies recombination, probably because these events require coinfection by viruses which normally replicate in distinct host organisms. This hypothesis was further supported by data from patients and co-workers, indicating that human retroviruses may be inefficiently recognized and packaged by the MLV assembly machinery [19]. However, in contrast with previous studies, recent data from our laboratory revealed unexpected in vivo interactions between distinct retroviral structures. Hereafter, we briefly describe and discuss our observations in an in vivo ovine model, providing evidence for in vivo recombination between a gibbon ape leukemia virus (GaLV)pseudotyped MLV-derived retroviral vector and bovine leukemia virus, a leukemogenic complex retrovirus that infects cattle and sheep. The ovine in vivo experimental approach described here relies on the possibility to easily detect novel acquired biological properties resulting from recombination, thus providing a unique tool for studying the changes in integrated retroviral structures. Our observations address the biosafety issue associated with retroviral vector stability in vivo, a key parameter that may impact the therapeutic potential of retroviral vector-based gene therapy.

We have developed a retroviral vector-mediated gene transfer strategy in a model that was initially established for investigating bovine leukemia virus (BLV)-associated leukemia in sheep. BLV is a complex retrovirus closely related to the HTLV family of human retroviruses and the ovine leukemia model provides an excellent means for studying aspects of human complex retrovirus-induced diseases (reviewed in $[20,21]$ ). In this highly reproducible model of BLV-induced lymphoid tumors, viral infection can be monitored after one week postinoculation, following injection of either naked provirus or virus particles produced in tissue culture.

The lack of viral expression in the ovine tumors has made it difficult to identify the discrete mechanisms by which BLV can mediate leukemogenesis [22, 23]. Tax, the transactivating transcriptional activator of viral expression, is believed to initiate early events in the multistep process leading to full malignancy, but its expression is not required to maintain the transformed phenotype. To gain insight into the mechanisms that govern virus silencing in BLV-induced tumors, we have studied YR2 leukemic B cells that contain a single, monoclonally integrated, silent provirus, which cannot be reactivated by either in vitro stimulation, or in vivo injection of the tumor cells or the cloned proviral DNA in sheep. Sequence analysis of the YR2 proviral tax gene identified two G to A transitions responsible for $\mathrm{E}$ to $\mathrm{K}$ amino acid changes at positions 228 and 303 of the Tax protein (309 aa) resulting in a transactivation-deficient phenotype. (GaLV)pseudotyped pLTaxSN retroviral vector-mediated transfer of wild-type tax into YR2 cells [24] resulted in the production of BLV transcripts characterized by the parental mutations in tax, thus potentially leading to a defective provirus. Injection of sheep with transduced cells was then assayed to investigate the in vivo infectious potential of BLV particles induced by LTaxSN. YR2 $2_{\text {LTaxSN }}$ inoculation resulted in rapid seroconversion and lead to the in vivo rescue of replication-competent proviruses with unique chimeric tax genes, which arose from recombination between the transduced LTaxSN vector-derived wildtype tax and the YR2-derived tax sequences [25].

We furthermore found that while BLV-specific antibody titers rose over time in the majority of these inoculated animals, a limited number of sheep displayed weak and transient antibody responses that were no longer detectable three months postinoculation. Most interestingly, one of those seronegative animals converted to high serological titers 18 months postinoculation, indicating that recombinant infectious proviruses generated by recombination with retroviral vector sequences may survive and emerge long after the initial injection. Finally, recent observations in a YR2 $2_{\text {LTaxSN-injected leukemic sheep revealed }}$ that a unique chimeric Tax-mutated replication-deficient provirus was integrated in the malignant $\mathrm{B}$ cell clone while recombinant functional provirus had been consistently monitored over the aleukemic period (Van den Broeke, unpublished observations, 2001). This observation strongly supports the hypothesis that switching off expression of Tax, an essential contributor to the oncogenic potential of BLV, is linked with the onset of acute leukemia.

In terms of BLV-associated leukemogenesis, our data provided clear evidence for the role of strategicallylocated mutations in retrovirus tumor-associated silencing, stressing the relevance of viral immunosurveillance escape mechanisms, and thus recombination, in the onset of the tumor. Most importantly, in terms of viral vector biosafety, our in vivo experimental approach based on the inoculation of retroviral vector-transduced tumor cells (YR2 $2_{\text {LTaxSN }}$ ) in sheep raised important questions regarding the in vivo stability of retroviral vectors, providing clear evidence for the presence of chimeric proviruses, as well as the long-term storage and subsequent selection of recombinant retroviral genetic information. Of particular interest was the emergence, as monitored by the full seroconversion, of a functional BLV provirus after an 18month period of seronegative carrier state in one of our experimental sheep. This suggests that the in vivo generation of mosaic viruses is a dynamic process and that virus variants, generated by retroviral vector-mediated 
recombination, may be stored and persist in the infected individual prior to selection at the level of replication. Finally, our findings suggest that there is a potential link between the modification of an integrated proviral structure and the emergence of pathology. In our model, retroviruses face changing selective forces favoring either increased viral fitness during the productive stage of the infection, or virus silencing during the leukemic stage of the BLV-associated disease. Thus, the system is actively driving recombination, generating retroviruses with altered functional capacities to fulfil the novel in vivo requirements. Reactivation restores full virulence. Silencing is likely to facilitate escape from immune responses and leads to full-blown malignancy. To our knowledge, this is the first example of a direct deleterious in vivo effect associated with vector-mediated recombination. In this particular case, the mechanism by which the immune system targets and destroys developing malignancies, is evaded by the pre-malignant cell by downregulating its intrinsic immunogenicity through recombination-mediated virus silencing.

These in vivo observations using a sheep model break new ground in our understanding how retroviral vectors may have an impact on the course of a preestablished disease or reactivate dormant or endogenous viruses. Recombination may not only restore essential viral functions or provide selective advantages in a changing environment but also re-establish or enhance the pathogenic potential of the particular virus undergoing recombination, such as the silencing-associated leukemogenicity studied as a model in our project. Our study furthermore stresses the limitations associated with retroviral vector-mediated gene delivery using current vectors in a host population infected with retroviruses (HIV, HTLV-I) or genomes that harbor endogenous retroelements. Although the exact mechanisms leading to the generation of mosaic retroviruses remain uncertain, our observations in sheep illustrate the largely ignored potential risks associated with retroviral gene transfer in humans. Because of the enormous selective pressures exerted in vivo, even theoretically rare events may pose a realistic safety concern. Further advancement in retroviral vector-mediated gene therapy and development of safe strategies for gene delivery must deal with the risks of in vivo recombination, generation of mosaic retroviruses, and long-term storage of viral genetic information. Future research will probably need to focus more on the in vivo aspects of vector biosafety and stability, a major concern with possible serious consequences to the therapeutic potential of retroviral vector based gene therapy.

\section{ACKNOWLEDGMENTS}

Work from the authors' laboratory was supported by funds from the Fonds Médic, the Fonds National de la Recherche Scientifique, the Association Française Contre les Myopathies, and the Gene Vector Production Network.

\section{REFERENCES}

[1] Hu WS, Pathak VK. Design of retroviral vectors and helper cells for gene therapy. Pharmacol Rev. 2000; 52(4):493-511.

[2] Mansky LM. Retrovirus mutation rates and their role in genetic variation. J Gen Virol. 1998;79(pt 6):1337-1345.

[3] Bowman RR, Hu WS, Pathak VK. Relative rates of retroviral reverse transcriptase template switching during RNA- and DNA-dependent DNA synthesis. J Virol. 1998;72(6):5198-5206.

[4] Negroni M, Buc H. Mechanisms of retroviral recombination. Annu Rev Genet. 2001;35:275-302.

[5] Mikkelsen JG, Pedersen FS. Genetic reassortment and patch repair by recombination in retroviruses. J Biomed Sci. 2000;7(2):77-99.

[6] Wilson CA, Ng TH, Miller AE. Evaluation of recommendations for replication-competent retrovirus testing associated with use of retroviral vectors. Hum Gene Ther. 1997;8(7):869-874.

[7] Chen J, Reeves L, Cornetta K. Safety testing for replication-competent retrovirus associated with gibbon ape leukemia virus-pseudotyped retroviral vectors. Hum Gene Ther. 2001;12(1):61-70.

[8] Zufferey R, Dull T, Mandel RJ, et al. Self-inactivating lentivirus vector for safe and efficient in vivo gene delivery. J Virol. 1998;72(12):9873-9880.

[9] Dull T, Zufferey R, Kelly M, et al. A third-generation lentivirus vector with a conditional packaging system. J Virol. 1998;72(11):8463-8471.

[10] Sheridan PL, Bodner M, Lynn A, et al. Generation of retroviral packaging and producer cell lines for large-scale vector production and clinical application: improved safety and high titer. Mol Ther. 2000;2(3):262-275.

[11] Chong H, Starkey W, Vile RG. A replicationcompetent retrovirus arising from a split-function packaging cell line was generated by recombination events between the vector, one of the packaging constructs, and endogenous retroviral sequences. J Virol. 1998;72(4):2663-2670.

[12] Martinez I, Dornburg R. Partial reconstitution of a replication-competent retrovirus in helper cells with partial overlaps between vector and helper cell genomes. Hum Gene Ther. 1996;7(6):705-712.

[13] Reuss FU, Heber R, Ploss A, Berdel B. Amphotropic murine leukemia virus replication in human mammary epithelial cells and the formation of cytomegalovirus-promoter recombinants. Virology. 2001;291(1):91-100.

[14] Boeke JD, Stoye JP. Retrotransposons, endogenous retroviruses, and the evolution of retroelements. In: Coffin JM, Hughes SH, Varmus HE, eds. Retroviruses. Cold Spring Harbor, NY: Cold Spring Harbor Press; 1997:343-435.

[15] Wolgamot G, Bonham L, Miller AD. Sequence analysis of Mus dunni endogenous virus reveals a hybrid 
VL30/gibbon ape leukemia virus-like structure and a distinct envelope. J Virol. 1998;72(9):7459-7466.

[16] Wooley DP, Smith RA, Czajak S, Desrosiers RC. Direct demonstration of retroviral recombination in a rhesus monkey. J Virol. 1997;71(12):9650-9653.

[17] Stoye JP. Endogenous retroviruses: still active after all these years? Curr Biol. 2001;11(22):R914-R916.

[18] Rasmussen HB. Interactions between exogenous and endogenous retroviruses. J Biomed Sci. 1997;4(1):18.

[19] Patience C, Takeuchi Y, Cosset FL, Weiss RA. Packaging of endogenous retroviral sequences in retroviral vectors produced by murine and human packaging cells. J Virol. 1998;72(4):2671-2676.

[20] Burny A, Willems L, Callebaut I, et al. Bovine leukemia virus: biology and mode of transformation. In: Minson AC, Neil JC, McRae MA, eds. Viruses and Cancer. Cambridge, UK: Cambridge University Press; 1994:213-234.

[21] Willems L, Burny A, Collete D, et al. Genetic determinants of bovine leukemia virus pathogenesis. AIDS Res Hum Retroviruses. 2000;16(16):1787-1795.

[22] Van den Broeke A, Cleuter Y, Droogmans L, Burny A, Kettman R. Isolation and culture of B lymphoblastoid cell lines from bovine leukemia virusinduced tumors. In: Lefkovits Y, ed. Immunology Methods Manual. In Vitro Experimental Immunology in Sheep. London, UK: Academic Press; 1997:21272132.

[23] Van den Broeke A, Cleuter Y, Chen G, et al. Even transcriptionally competent proviruses are silent in bovine leukemia virus-induced sheep tumor cells. Proc Natl Acad Sci USA. 1988;85(23):9263-9267.

[24] Bagnis C, Chischportich C, Imbert AM, Van den Broeke A, Cornet V, Mannoni P. Efficiency of retroviral transduction into hematopoietic cells by cocultivation procedure does not correlate with viral titer. Cancer Gene Ther. 1997;4(1):5-8.

[25] Van den Broeke A, Bagnis C, Ciesiolka M, et al. In vivo rescue of a silent tax-deficient bovine leukemia virus from a tumor-derived ovine B-cell line by recombination with a retrovirally transduced wildtype tax gene. J Virol. 1999;73(2):1054-1065.

\footnotetext{
* Corresponding author. E-mail: anne_vandenbroeke@compuserve.com Fax: +32 2541 3453; Tel: +32 25413739
} 

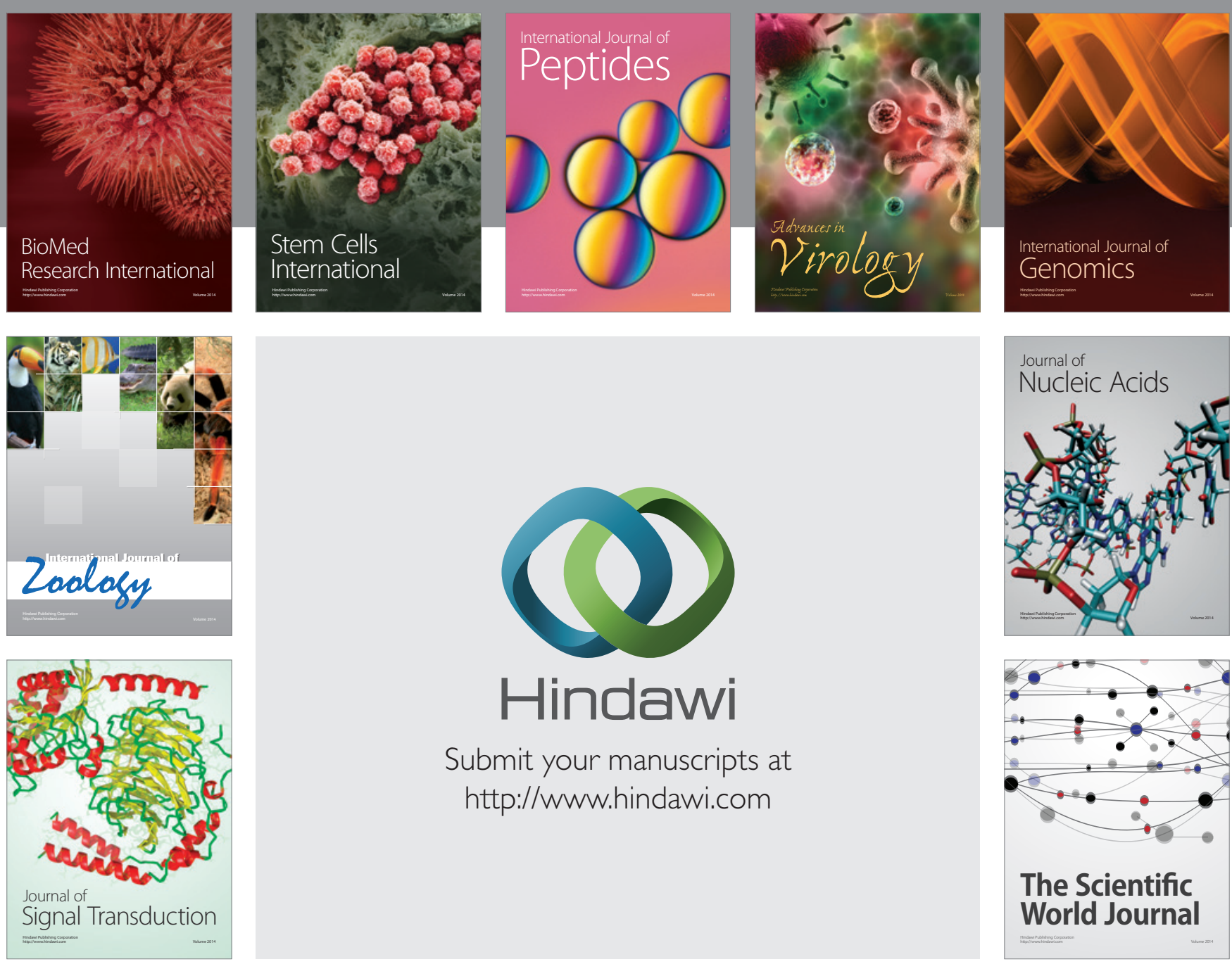

Submit your manuscripts at

http://www.hindawi.com
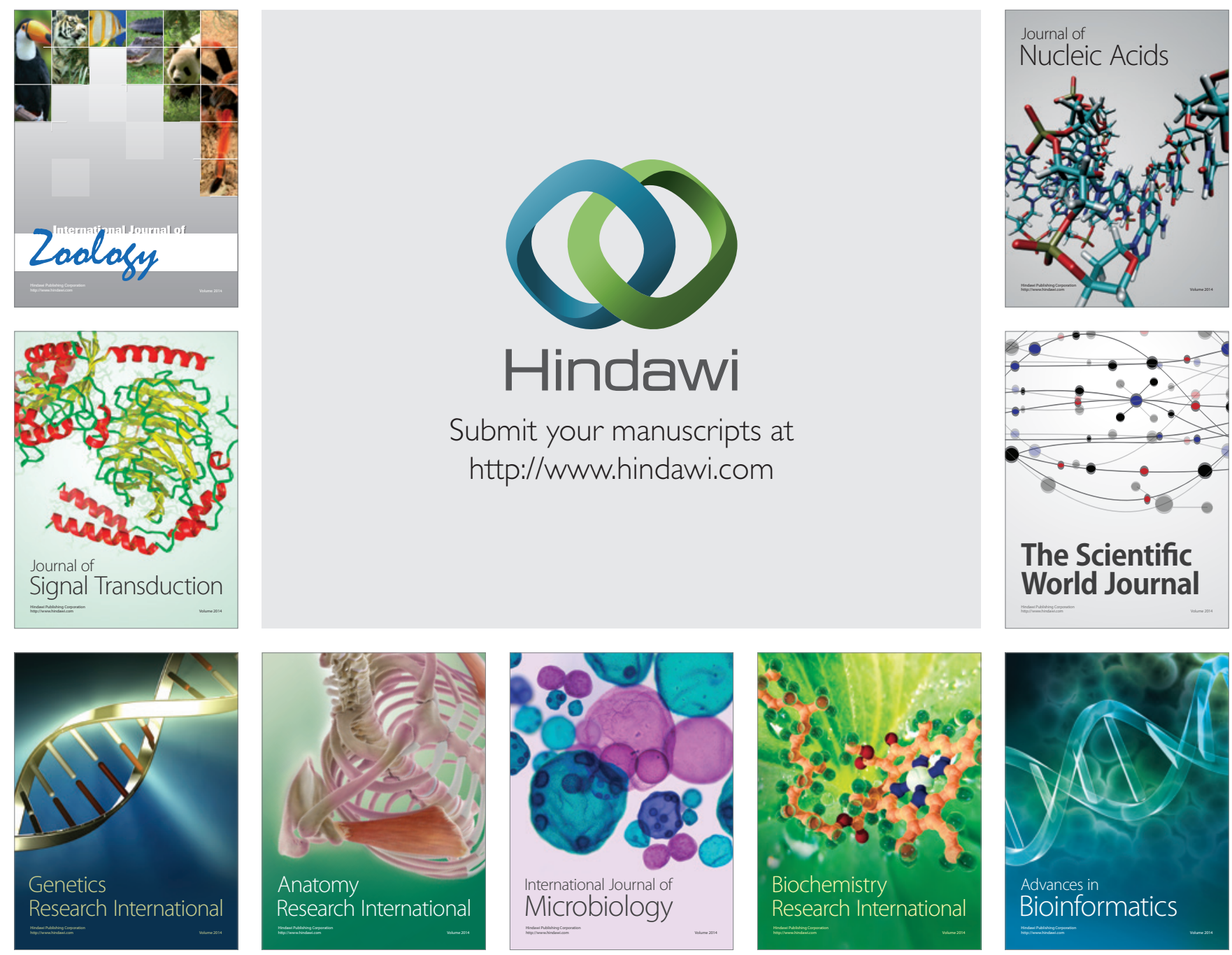

The Scientific World Journal
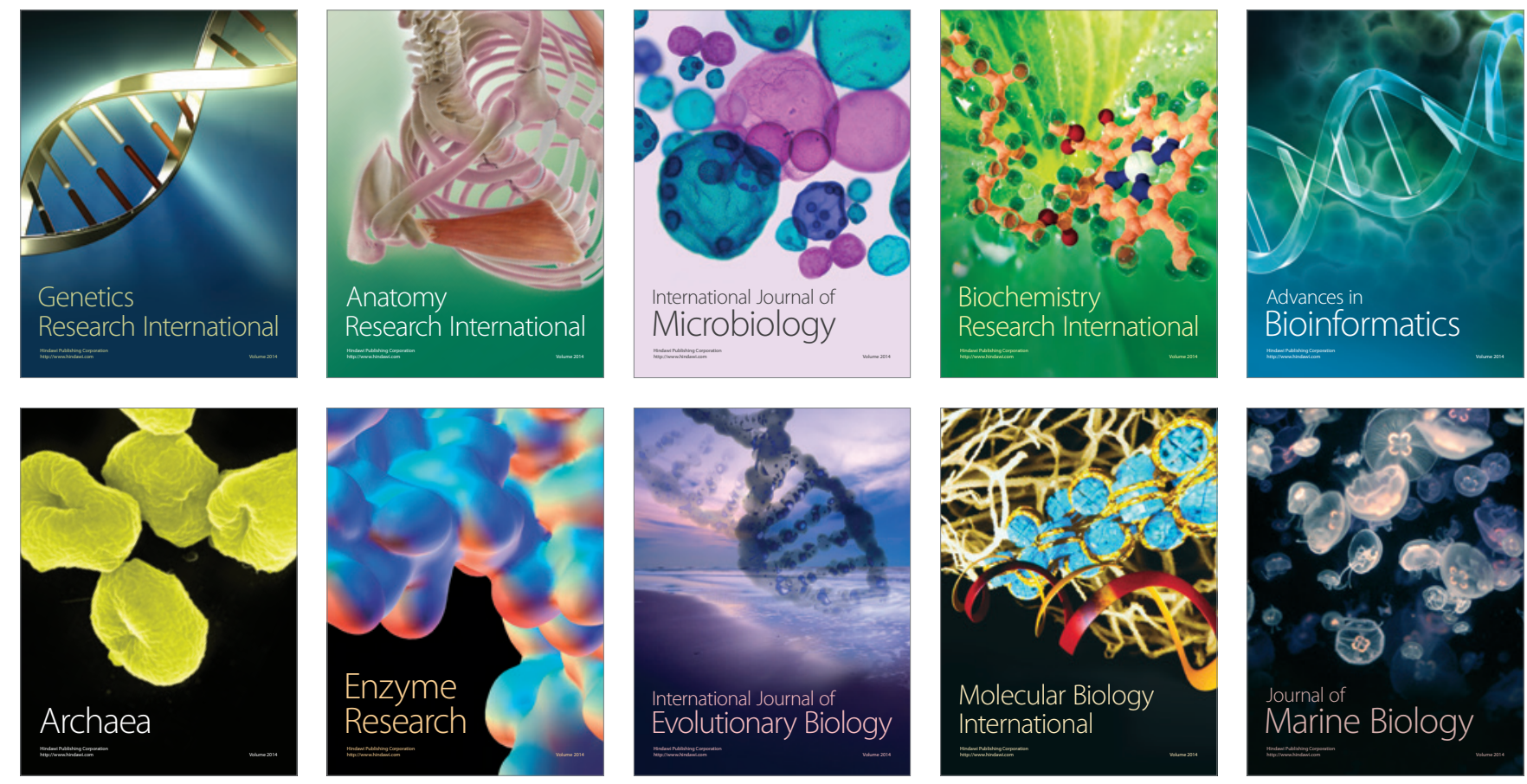